\title{
G $\begin{array}{ll}\text { Research Square } & \text { They should not be considered conclusive, used to inform clinical practice, } \\ \text { or referenced by the media as validated information. }\end{array}$
}

\section{Adipose-derived stem cells combined with platelet- rich plasma enhance wound healing in a rat model of full-thickness skin defects}

\section{Xuejun Ni}

the First Affiliated Hospital of Fujian Medical University

\section{Xiuying Shan}

the First Affiliated Hospital of Fujian Medical University

\section{Lili Xu}

the First Affiliated Hospital of Fujian Medical University

\section{Wenjun Yu}

the First Affiliated Hospital of Fujian Medical University

\section{Mingliang Zhang}

the First Affiliated Hospital of Fujian Medical University

Chen Lei

the First Affiliated Hospital of Fujian Medical University

Nating Xu

the First Affiliated Hospital of Fujian Medical University

Junyu Lin

the First Affiliated Hospital of Fujian Medical University

Biao Wang ( $1812166371 @ q q . c o m$ )

the First Affiliated Hospital of Fujian Medical University

\section{Research}

Keywords: Adipose-derived stem cell, Platelet-rich plasma, Diabetic wound healing, Neovascularization.

Posted Date: February 4th, 2021

DOI: https://doi.org/10.21203/rs.3.rs-83182/v2

License: (c) (1) This work is licensed under a Creative Commons Attribution 4.0 International License. Read Full License

Version of Record: A version of this preprint was published at Stem Cell Research \& Therapy on April 6th, 2021. See the published version at https://doi.org/10.1186/s13287-021-02257-1. 


\section{Abstract}

Background: Wound healing is impaired in patients with diabetes due to the multifactorial etiology of the disease, which limits the therapeutic efficacy of various approaches. This study hypothesizes that the combination of adipose-derived stem cells (ADSCs) and platelet-rich plasma (PRP) might achieve optimally efficient diabetic wound healing.

Methods: ADSCs were isolated from the adipose tissues of Sprague-Dawley (SD) rats. PRP was prepared by using a two-step centrifugation technique. A diabetic wound model was established on the backs of SD rats to evaluate the effect of ADSCs incorporated into PRP. Hematoxylin and eosin staining, immunofluorescence and immunohistochemistry were performed to observe the changes in neovascularization. ELISA and Western blot were utilized to detect the angiogenesis-related protein expression levels. The proliferation of endothelial cells was assessed by the MTS assay.

Results: ADSCs incorporated into PRP induced a higher wound closure rate than ADSCs, PRP and negative control. The expression levels of VEGF, p-STAT3 and SDF-1 in the ADSC-PRP group were higher than those in the other groups. Moreover, the proliferation of endothelial cells was strongly stimulated by treatment with the combination of ADSC-conditioned medium (ADSC-CM) and PRP.

Conclusions: PRP enhanced diabetic wound healing induced by ADSCs, and its promoting effect involved neovascularization.

\section{Background}

Wound healing is a sequential and overlapping biological process that includes hemostasis, inflammation, growth, reepithelialization, and remodeling. It involves spatial and temporal coordination of multiple cell types and signaling pathways[1]. Accordingly, some injuries or disease states, such as diabetes, interrupt the normal wound healing progress and ultimately result in chronic or nonhealing wounds. Impairment of physiological activities in diabetes delays wound healing and ultimately results in chronic wounds. As the worldwide prevalence of diabetes mellitus and the life expectancy of diabetic patients have increased, the incidence of diabetic-related chronic wounds, which have become a serious public health problem, has also been amplified[2]. The development of efficient and accessible treatments for chronic wound healing is necessary.

Stem cell therapy might be a promising treatment option for chronic wounds due to the ability of stem cells to self-renew and differentiate into numerous types of cells[3]. It has been proven that stem cell therapy can contribute to diabetic wounds and other diseases[4-6]. Compared with other types of stem cells, adipose-derived stem cells (ADSCs) have specific advantages and fewer limitations; they are abundant and easily harvested, can be delivered via a minimally invasive procedure, are associated with fewer ethical issues and have less risk of inducing tumorigenicity and the host immune response[7, 8]. A large number of studies have verified the therapeutic efficacy of ADSCs for wound healing[9-13]. However, 
the repair function of ADSCs is inherently restricted by the hostile microenvironment of chronic wounds due to their complex mechanism[14, 15].

Platelet-rich plasma (PRP), which provides an abundance of nutrients and offers a suitable microenvironment for ADSCs to promote proliferation and migration, is a good candidate for enhancing the wound healing effect of ADSCs due to its unique composition[16, 17]. PRP is an autologous product of blood plasma with a high platelet concentration and contains various growth factors and cytokines, such as platelet-derived growth factor (PDGF), transforming growth factor (TGF), vascular endothelial growth factor (VEGF) and insulin-like growth factor (IGF)[18]. Although more evidence of the efficacy of PRP alone as a treatment for wound healing is needed, the positive impact of PRP on chronic wounds has been proven in many studies[19]. More importantly, PRP can promote the proliferation and migration of ADSCs, which indicates that it has a potential synergistic regenerative effect on chronic wounds[2022].

To our knowledge, relevant research on the combination of ADSCs and PRP for the treatment of wound healing is very limited. Based on the above results, we believe that the application of ADSCs in combination with PRP can improve the efficiency of wound healing. We established a wound model in diabetic rats and evaluated the synergistic effect of ADSCs and PRP on wound healing. The results showed that compared with the negative control, ADSCs, and PRP, the combination of ADSCs and PRP, which accelerated angiogenesis, greatly promoted wound healing in diabetic rats. The results of our study extend our understanding of the mechanism underlying the promoting effect of ADSCs and PRP on wound healing and might provide a constructive foundation for further research.

\section{Methods}

\section{Isolation and culture of ADSCs}

Male Sprague-Dawley (SD) rats were purchased from the Experimental Animals Centre of Fujian Medical University. All animal experiments were approved by the Animal Ethics Committee of the First Affiliated Hospital of Fujian Medical University. Rat ADSCs were isolated from the inguinal fat tissue of SD rats (2024 weeks old, male) according to a previously reported method[23]. In brief, the inguinal fat tissues were isolated and washed with phosphate-buffered saline (PBS; Invitrogen, Carlsbad, CA). Then, the tissues were minced and digested with $0.1 \%$ collagenase type I (Sigma-Aldrich, St. Louis, MO) for 1 hour at $37^{\circ} \mathrm{C}$. Enzyme activity was neutralized with low-glucose Dulbecco's modified Eagle medium (LG-DMEM; HyClone, Logan, UT). After centrifugation, the pellet was resuspended in LG-DMEM supplemented with $10 \%$ fetal bovine serum (FBS; Gibco, Carlsbad, CA). The isolated cells were maintained in tissue culture flasks with culture medium at $37^{\circ} \mathrm{C}$ in a $5 \% \mathrm{CO}_{2}$ incubator.

\section{Confirmation of mesenchymal stem cell (MSC) characteristics}

Immunophenotyping was performed by flow cytometry. Briefly, ADSCs were trypsinized, centrifuged, and incubated at $4^{\circ} \mathrm{C}$ for 30 min with fluorescein isothiocyanate (FITC)-labeled anti-CD34, FITC-labeled anti- 
CD45, FITC-labeled anti-CD90, and FITC-labeled anti-CD29 antibodies (BioLegend, San Diego, CA). The cells were analyzed using a FACSCalibur cytometer (Becton Dickinson, San Diego, CA), and FlowJo software V10 was used to generate histograms. The multilineage differentiation of ADSCs was analyzed after 21 days of induction. For adipogenic induction, ADSCs were cultured with medium containing adipogenic reagents, and the culture medium was replaced every 2-3 days. After 21 days of incubation, oil red $\mathrm{O}$ staining was performed to confirm the formation of lipid droplets. ADSCs were also cultured with medium containing osteogenic reagents for osteogenic induction. The presence of calcium nodules was evaluated by Alizarin red staining.

\section{Preparation of PRP}

PRP was obtained from blood drawn from SD rats by cardiac puncture using a two-step centrifugation process according to a previously reported method[24]. The whole blood was centrifuged at $160 \times \mathrm{g}$ for $20 \mathrm{~min}$ to separate the plasma. The plasma fraction was centrifuged again at $400 \times \mathrm{g}$ for $15 \mathrm{~min}$ to separate the PRP. A thrombin activator (500 $\mathrm{U}$ bovine thrombin in $1 \mathrm{ml} 10 \%$ calcium chloride) was added to activate the PRP.

\section{Transwell migration assay}

To examine ADSCs migration induced by different concentrations of PRP, Transwell Permeable Supports (\#3422, CORNING, NY, USA) were applied. ADSCs were placed in the upper chamber of a Transwell plate, while different concentrations of PRP were added to the lower chamber. The concentration gradient of PRP was as follows: $0 \%, 5 \%, 10 \%, 20 \%, 30 \%, 40 \%, 80 \%$ and $100 \%$. LG-DMEM containing $10 \%$ FBS was added as a control. After incubation for 24 hours at $37^{\circ} \mathrm{C}$, the migrated ADSCs were fixed with $4 \%$ paraformaldehyde and then stained using crystal violet. Finally, the stained cells were photographed under a microscope for counting.

\section{Diabetic wound model and wound healing assessment}

Female SD rats were injected with streptozotocin (Sigma-Aldrich) to induce diabetes. The rats that exhibited hyperglycemia within 1 week after injection were confirmed as diabetic rats, and the rats were then anesthetized by intraperitoneal injection of $10 \%$ chloral hydrate. The dorsal hair was removed, two circular wounds (diameter, $10 \mathrm{~mm}$ ) were created on the dorsum of rats, and silicone rings were sutured around the wounds to prevent contracture. A total of $0.4 \mathrm{ml} \mathrm{PBS} \mathrm{(control),} 0.4 \mathrm{ml} \mathrm{ADSCs}\left(1 \times 10^{6} / \mathrm{ml}\right), 0.4$ $\mathrm{ml} \mathrm{PRP}$, or $0.4 \mathrm{ml} \mathrm{ADSCs}\left(1 \times 10^{6} / \mathrm{ml}\right)+$ PRP were administered to each wound of the rats from each group. Wound size was measured from photographs $0,1,3,7,10$ and 14 days after the operation. The experimental design is shown in Figure 1.

\section{Histological observation}

The wound tissues were fixed in $4 \%$ paraformaldehyde overnight at $4^{\circ} \mathrm{C}$. After being washed with PBS, they were dehydrated in a graded ethanol series (30-100\%) and embedded in paraffin. Sections (4- to 6- 
mm-thick) were prepared from the paraffin-embedded wound tissues and then stained with hematoxylin and eosin as well as Masson's trichrome.

\section{Immunohistochemistry and immunofluorescence}

The sections were prepared as described above. For immunohistochemistry, sections were deparaffinized in xylene and dehydrated through a graded series of alcohol. High-pressure antigen retrieval was performed with citrate antigen repair solution and then incubated in $3 \%$ hydrogen peroxide at room temperature for $20 \mathrm{~min}$. The slices were incubated with primary rabbit polyclonal antibodies against stromal cell-derived factor-1 (SDF-1, 1:200 dilution, Abcam, Cambridge, MA) at $4^{\circ} \mathrm{C}$ overnight and then incubated with a horseradish peroxidase-labeled secondary antibody at $37^{\circ} \mathrm{C}$ for $30 \mathrm{~min}$. Next, 3,3'-diaminobenzidine (DAB) was added at room temperature for $10 \mathrm{~min}$, and the slices were then stained with hematoxylin at room temperature for 2 min. Finally, the slices were gently washed with deionized water, dehydrated in gradient alcohol solutions, mounted with neutral balsam, and observed using an optical microscope. Anti-CD31 and anti-CD34 antibodies (1:200 dilution, Abcam, Cambridge, MA) were used for immunofluorescence. As for immunohistochemistry, the slices were incubated with the abovementioned primary antibodies at $4^{\circ} \mathrm{C}$ overnight and then incubated with a secondary antibody at $37^{\circ} \mathrm{C}$ for 1 hour. The slices were finally stained with 4',6-diamidino-2-phenylindole (DAPI, Invitrogen, Carlsbad, CA) and observed under a Zeiss LSM 700 confocal fluorescence microscope.

\section{Enzyme-linked immunosorbent assay (ELISA)}

To measure the concentration of VEGF in wound tissues, the specimens were ground, trypsinized and centrifuged to prepare protein extracts. The concentration of VEGF was then determined by an ELISA kit (R\&D Systems, Minneapolis, MN) according to the manufacturer's instructions. Briefly, 96-well plates were coated with an VEGF antibody. Two hundred microliters of standard, control, or sample was added to consecutive wells and incubated at room temperature for 2 hours. Each well was then washed 3 times with wash buffer, and $200 \mu \mathrm{l} \mathrm{VEGF} \mathrm{conjugate} \mathrm{was} \mathrm{added} \mathrm{to} \mathrm{each} \mathrm{well.} \mathrm{After} \mathrm{incubation} \mathrm{at} \mathrm{room}$ temperature for 2 hours, $200 \mu$ l substrate solution was added to each well, and the samples were incubated for $20 \mathrm{~min}$ at room temperature. The concentrations were then determined at $450 \mathrm{~nm}$ using a microplate reader.

\section{Western blot}

Protein concentration was quantified using the BCA method. Western blotting was carried out according to the standard protocols. Proteins were separated by $10 \%$ SDS-PAGE and transferred to a PVDF membrane. The membrane containing transferred proteins was blocked with $5 \%$ skim milk in TBS at room temperature for $1 \mathrm{~h}$ and with 1:1000 dilutions of anti-p-STAT3 (Abcam, Cambridge, MA) overnight at $4^{\circ} \mathrm{C}$. The next day, the membranes were incubated with secondary antibody at a 1:1000 dilution for $2 \mathrm{~h}$ at room temperature after washed with TBST for three times. Chemiluminescence detection was performed using the ECL reagent (Bio-Rad Laboratories). The strength of the signal for each protein was determined based on the corresponding band intensity of the scanned image. 


\section{Endothelial cell culture and the MTS assay}

Rat dermis microvascular endothelial cells were isolated from dermal microvasculature and cultured as previously described[25]. To evaluate the proliferation of viable endothelial cells, a total of $5 \times 10^{3}$ endothelial cells per well were cultured in 96-well plates in $100 \mu \mathrm{l}$ medium containing ADSCs culture medium (ADSC-CM), $20 \%$ PRP, ADSC-CM+20\% PRP or $10 \%$ FBS as the control. Twenty microliters of 3(4,5-dimethylthiazol-2-yl)-5-(3-carboxymethoxyphenyl)-2-(4-sulfophenyl)-2H-tetrazolium (MTS, Promega, Madison, $\mathrm{WI})$ was added to each well. After incubation for 4 hours, absorption values were measured at a wavelength of $490 \mathrm{~nm}$ using a microplate reader.

\section{Statistical analysis}

Statistical analysis was performed with IBM SPSS Statistics 25. All data are expressed as the mean \pm SEM. One-way analysis of variance for multiple comparisons with Tukey's post hoc test was used to determine statistical significance, which was defined by a P-value $<0.05$.

\section{Results}

\section{Characterization and identification of ADSCs}

The MSC characteristics of isolated ADSCs were first confirmed based on the expression of two MSC surface antigens (CD29 and CD90) and two endothelial progenitor cell markers (CD34 and CD45), as measured by flow cytometry. Positive expression of CD29 and CD90 and negative expression of CD34 and CD45 were detected, validating the identity of the ADSCs used in this study (Figure 2A). In addition, we tested the multilineage differentiation potential of ADSCs. Oil red 0 staining demonstrated that lipid droplet formation was induced by adipogenic medium in ADSCs after 21 days, and Alizarin red staining showed calcium nodules in ADSCs after 21 days of induction with osteogenic medium (Figure 2B). Thus, the ADSCs utilized in this study were successfully isolated, and their multilineage potential was confirmed.

\section{Combined treatment with ADSCs and PRP improved diabetic wound healing}

To determine the optimal concentration of PRP for combination with ADSCs, various concentrations of PRP were placed in the lower chamber of a Transwell plate to attract ADSCs cultured in the upper chamber. We found that $20 \%$ PRP induced the greatest migration of ADSCs, and this concentration of PRP was applied in this study (Figure 2C). A diabetic rat model was successfully established, and fullthickness skin wounds were created on the backs of the model rats. Forty rats were randomly divided into 4 groups and injected with PBS, ADSCs, PRP or ADSC-PRP. The areas of the cutaneous wounds were monitored every 3-4 days, and we found that compared with PBS, the application of ADSCs, PRP or ADSC-PRP significantly promoted wound closure (Figure 3A). It was obvious that the ADSC-PRP group exhibited that highest wound closure rate (Figure 3B). Wound reepithelialization and matureness were assessed 14 days postinjury; compared to the other groups, the ADSC-PRP group exhibited a thicker 
epidermis and increased appendages in the dermis (Figure 3C). The wounds injected with ADSC-PRP presented well-organized collagen deposition in the dermis, as determined by Masson's trichrome staining (Figure 3D).

\section{Combined treatment with ADSCs and PRP promoted new blood vessel formation in diabetic wounds}

We next explored early angiogenesis by hematoxylin and eosin staining and observed increased blood vessel formation in the wounds of the ADSC-PRP group compared to those of the PBS, ADSCs and PRP groups (Figure 4A). We further evaluated the expression of the vessel markers CD34, CD31, and a-SMA by immunohistochemistry. The wounds of the ADSC-PRP group exhibited the highest expression of CD34 and CD31, which indicated that ADSC-PRP treatment induced more new blood vessel formation. However, there was no significant difference in a-SMA expression among the groups (Figure 4B). Taken together, our results suggested ADSC-PRP improved diabetic wound healing by promoting neovascularization.

\section{Combined treatment with ADSCs and PRP improved diabetic wound healing, and these improvements were related to angiogenesis and vasculogenesis}

Angiogenesis and vasculogenesis are two types of neovascularization. We found that the expression of the proangiogenic factor VEGF was higher in the diabetic wounds of the ADSC-PRP group compared with those of the other groups, which demonstrated that ADSC-PRP stimulated angiogenesis (Figure 5A). Moreover, we analyzed the proliferation capacity of endothelial cells cultured with PBS, ADSC-CM, PRP or a combination of ADSC-CM and PRP. We found that compared to the other treatments, the combination of ADSC-CM with PRP more strongly promoted the proliferation of endothelial cells. More importantly, this enhancement of endothelial cell proliferation was retained when high-glucose medium was added (Figure 5B). Western blot analysis was used to determine the effect of ADSC-PRP on angiogenesisassociated protein. The ADSC-PRP group significantly up-regulated the expression of p-STAT3 after 7 days of treatment compared with the other groups (Figure $5 \mathrm{C}$ ). We finally evaluated vasculogenesis by detecting the expression of SDF-1 by immunohistochemistry. The results showed that combined treatment with ADSCs and PRP induced a greater increase in the protein expression of SDF-1 than the other treatments (Figure 5D).

\section{Discussion}

Wound healing is a complicated but highly regulated biological process. Many factors can disturb the well-organized process, resulting in chronic or nonhealing wounds. Due to its molecular pathogenesis, diabetes is a factor that can disrupt the process of wound healing and lead to the development of a chronic or nonhealing wound. A series of studies have uncovered the probable pathophysiology of diabetic wounds, which includes decreased growth factor production, impaired angiogenesis and defective cellular functions in the impaired wound[26]. The current conventional treatments, such as application of dressings, negative pressure therapy, autologous skin graft and hyperbaric oxygen therapy, have several limitations and moderate efficacy[7]. Developing an innovative and more efficient treatment is urgent. 
Stem cell therapy has become a promising approach to promote wound healing. Stem cells can not only secrete various growth factors but also directly transdifferentiate into vascular components or skin cells to correct the pathogenesis of diabetic wounds[6]. The clinical use of human allogenic ADSCs transplant had been used for many years, while the safety and efficacy were confirmed in osteoarthritis, acute ischemic stroke, myocardial infarction and so on[27]. ADSCs even have the possibility to be used in patients with COVID-19 pneumonia[28]. For chronic wounds, several mechanisms of ADSCs were described in each process of wound healing, including the reduction of inflammation, induction of angiogenesis, and promotion of fibroblast and keratinocyte growth[29].

PRP is another effective agent for wound healing because it contains abundant growth factors and other substances that can restore growth factors in diabetic wounds. The cellular processes, such as chemotaxis, cell differentiation and angiogenesis, and several target cells in wound healing were stimulated by PRP[30, 31]. The antiapoptotic effect of PRP through the activation of the Bcl-2 protein and Akt signaling was a contributing factor to improve wound healing by stimulating hair growth[32]. Recently, the combined use with PRP and hyaluronic acid as a bio-functionalized scaffold showed many advantages for wound healing[30]. Similarly, PRP can act as a biomaterial scaffold for ADSCs, and furthermore, it can induce the development of angiogenesis for delivering proper nutrient and oxygen levels to grafted ADSCs[33]. Therefore, combining ADSCs with PRP might be an optimal therapeutic strategy for enhancing the healing of chronic/nonhealing wounds.

In this study, we established a wound model in diabetic rats to explore the treatment efficacy of a combination of ADSCs and PRP. We found that the ADSC-PRP group had a higher wound closure rate than the control, PRP and ADSCs groups. Treatment with ADSC-PRP induced complete closure of diabetic wounds after 10 days, while the other treatments did not, indicating the synergistic effects of ADSCs and PRP. We observed histological changes in the wounds to evaluate the function of ADSCs and PRP and found that thicker epidermis and more well-organized collagen deposition were presented in the dermis. The stimulatory effects of ADSCs and PRP on proliferation and migration of fibroblasts and keratinocytes revealed the mechanism of the above phenomena[34].

In addition, more vascular structures appeared in the wounds of the ADSC-PRP group. New vessel formation, which allows delivery of nutrients and maintenance of oxygen homeostasis, is required for efficient wound healing. However, the crucial process of wound healing is impaired in diabetes mellitus. Diabetic hyperglycemia can impede angiogenesis by perturbing the balance of vessel growth and inducing the dysfunction of endothelial cells[35]. Defective new blood vessel formation is a major contributor to chronic/nonhealing wounds caused by diabetes. The ADSC-PRP-induced restoration of vessel formation in chronic wounds showed the potential to correct the abnormal healing process. We next verified the promoting effect of ADSC-PRP on new vessel formation by using immunofluorescence and found that the expression of the vascular markers CD31 and CD34 in the wounds was highest in the ADSC-PRP group. These results suggest that the therapeutic effect of ADSC-PRP is related to neovascularization in diabetic wounds. 
An adequate newly developed vascular system is essential for wound healing. Angiogenesis is the primary method by which new blood vessels are developed and is tightly controlled by the balance of proangiogenic and antiangiogenic factors[36]. VEGF is one of the most important proangiogenic factors and can stimulate multiple steps of angiogenesis, including vasodilation, basement membrane degradation, endothelial cell migration and proliferation[37]. The VEGF content in wounds was reduced in diabetic wounds compared to normal wounds, and this reduction in VEGF expression ultimately impaired angiogenesis[38]. Interestingly, we found that the combined application of ADSCs and PRP elevated the level of VEGF in diabetic wounds. The activation of endothelial cells in angiogenesis enhanced the capillary structure. Therefore, we speculated that ADSC-PRP also has a positive effect on the proliferation of endothelial cells. The paracrine activity of ADSCs mediated by ADSC-CM is an important way in which ADSCs interact with other cells in the extracellular microenvironment. We used ADSC-CM, PRP or both to stimulate the growth of endothelial cells in vitro and found that the combination of the two treatments had the strongest effect on increasing the proliferation of endothelial cells. The stimulation of endothelial cell proliferation also persisted under high-glucose conditions. This phenomenon might partly explain the increased vessel formation in diabetic wounds treated with ADSC-PRP. It has been established that the induction of VEGF expression is regulated by active STAT3 in the spectrum of physiological and pathological conditions[39]. So we detected the p-STAT3 expression in the diabetic wound and found ADSC-PRP could increase the expression of p-STAT3. Therefore, it is reasonable to presume that ADSCPRP might enhance angiogenesis through STAT3/VEGF axis. It is also worth noting that VEGF is not limited to accelerating angiogenesis but also affects other cells, such as fibroblasts and keratinocytes[40], to contribute to wound healing.

In addition to angiogenesis, vasculogenesis also contributes to neovascularization. When injury occurs, endothelial progenitor cells (EPCs) are mobilized from the bone marrow into the bloodstream and then recruited to the hypoxic environment of the wound. The above processes, called EPC mobilization and homing, are impaired in patients with diabetes[26]. The mobilization of EPCs could be partly stimulated by hyperoxia, but the inducer of EPC homing, SDF-1, is indeed decreased in patients with diabetes[41]. Gallagher found that SDF-1 injection into diabetic wounds partially reverses the defect in EPC recruitment and ultimately enhances diabetic wound healing[41]. We were surprised to find that SDF-1 expressed in diabetic wounds was restored by ADSC-PRP treatment.

Improvements in diabetic wound healing are urgently needed. Here, we propose that the combined application of ADSCs and PRP can enhance diabetic wound healing through neovascularization and is therefore an optimal treatment for diabetic wounds (Figure 6).

\section{Conclusions}

In summary, this study reveals that the combination of ADSCs and PRP can optimally enhance diabetic wound healing. The underlying mechanism involves neovascularization. This study provides an innovative collaborative scenario and a strong foundation for further treatment of wound healing. 


\section{Abbreviations}

ADSCs: adipose-derived stem cells; PRP: platelet-rich plasma; PDGF: platelet-derived growth factor; TGF: transforming growth factor; VEGF: vascular endothelial growth factor; IGF: insulin-like growth factor; SD: Sprague-Dawley; PBS: phosphate-buffered saline; LG-DMEM: low-glucose Dulbecco's modified Eagle medium; FBS: fetal bovine serum; FITC: fluorescein isothiocyanate; SDF-1: stromal cell-derived factor-1; DAB: 3,3'-diaminobenzidine; DAPI: 6-diamidino-2-phenylindole; ELISA: enzyme-linked immunosorbent assay; ADSC-CM: adipose-derived stem cell culture medium; MTS: 3-(4,5-dimethylthiazol-2-yl)-5-(3carboxymethoxyphenyl)-2-(4-sulfophenyl)-2H-tetrazolium; MSC: mesenchymal stem cell; EPCs: endothelial progenitor cells.

\section{Declarations}

\section{Ethics approval and consent to participate}

All animal experiments were approved by the Animal Ethics Committee of the First Affiliated Hospital of Fujian Medical University.

\section{Consent for publication}

Not applicable.

\section{Availability of data and materials}

Not applicable.

\section{Competing interests}

The authors declare that they have no competing interests.

\section{Funding}

This study was funded by the Scientific Research Foundation of National Health

Planning Scientific Research Foundation-Joint Research Projects of Fujian Provincial Health and Education (2019-WJ-09); Science and Technology Innovation Joint Fund Project of Fujian Province (2019Y9114) and the Start-up Foundation of Fujian Medical University (2019QH1091).

\section{Authors' contributions}


$\mathrm{XN}$ and XS performed the experiments, analyzed the data, and wrote the manuscript; BW reviewed and edited the manuscript; and LX, WY, MZ, CL, NX and JL researched the data. All authors read and approved the final manuscript.

\section{Acknowledgments}

We deeply appreciate the assistance of Dr. Hongteng Xie and the support of the laboratory of the College of Life Sciences of Fujian Normal University.

\section{References}

1. Rodrigues M, Kosaric N, Bonham CA, Gurtner GC. Wound Healing: A Cellular Perspective. Physiol Rev. 2019;99(1):665-706.

2. Zhang P, Lu J, Jing Y, Tang S, Zhu D, Bi Y. Global epidemiology of diabetic foot ulceration: a systematic review and meta-analysis (dagger). Ann Med. 2017;49(2):106-16.

3. Marquez-Curtis LA, Janowska-Wieczorek A, McGann LE, Elliott JA. Mesenchymal stromal cells derived from various tissues: Biological, clinical and cryopreservation aspects. Cryobiology. 2015;71(2):181-97.

4. Tabatabaei Qomi R, Sheykhhasan M. Adipose-derived stromal cell in regenerative medicine: A review. World journal of stem cells. 2017;9(8):107-17.

5. Lee DE, Ayoub N, Agrawal DK. Mesenchymal stem cells and cutaneous wound healing: novel methods to increase cell delivery and therapeutic efficacy. Stem Cell Res Ther. 2016;7:37.

6. Yang M, Sheng L, Zhang TR, Li Q. Stem cell therapy for lower extremity diabetic ulcers: where do we stand? BioMed research international. 2013;2013:462179.

7. Hassanshahi A, Hassanshahi M, Khabbazi S, Hosseini-Khah Z, Peymanfar Y, Ghalamkari S, et al. Adipose-derived stem cells for wound healing. 2019;234(6):7903-14.

8. Frese L, Dijkman PE, Hoerstrup SP. Adipose Tissue-Derived Stem Cells in Regenerative Medicine. Transfus Med Hemother. 2016;43(4):268-74.

9. Ebrahimian TG, Pouzoulet F, Squiban C, Buard V, Andre M, Cousin B, et al. Cell therapy based on adipose tissue-derived stromal cells promotes physiological and pathological wound healing. Arterioscler Thromb Vasc Biol. 2009;29(4):503-10.

10. Shingyochi Y, Orbay H, Mizuno H. Adipose-derived stem cells for wound repair and regeneration. Expert Opin Biol Ther. 2015;15(9):1285-92.

11. Moon KM, Park YH, Lee JS, Chae YB, Kim MM, Kim DS, et al. The effect of secretory factors of adipose-derived stem cells on human keratinocytes. International journal of molecular sciences. 2012;13(1):1239-57.

12. Hur W, Lee HY, Min HS, Wufuer M, Lee CW, Hur JA, et al. Regeneration of full-thickness skin defects by differentiated adipose-derived stem cells into fibroblast-like cells by fibroblast-conditioned medium. Stem Cell Res Ther. 2017;8(1):92. 
13. Yu J, Wang MY, Tai HC, Cheng NC. Cell sheet composed of adipose-derived stem cells demonstrates enhanced skin wound healing with reduced scar formation. Acta Biomater. 2018;77:191-200.

14. Koenen P, Spanholtz TA, Maegele M, Sturmer E, Brockamp T, Neugebauer E, et al. Acute and chronic wound fluids inversely influence adipose-derived stem cell function: molecular insights into impaired wound healing. International wound journal. 2015;12(1):10-6.

15. Ferrer-Lorente R, Bejar MT, Tous M, Vilahur G, Badimon L. Systems biology approach to identify alterations in the stem cell reservoir of subcutaneous adipose tissue in a rat model of diabetes: effects on differentiation potential and function. Diabetologia. 2014;57(1):246-56.

16. Zhang L, Zhang B, Liao B, Yuan S, Liu Y, Liao Z, et al. Platelet-rich plasma in combination with adipose-derived stem cells promotes skin wound healing through activating Rho GTPase-mediated signaling pathway. American journal of translational research. 2019;11(7):4100-12.

17. Kocaoemer A, Kern S, Kluter H, Bieback K. Human AB serum and thrombin-activated platelet-rich plasma are suitable alternatives to fetal calf serum for the expansion of mesenchymal stem cells from adipose tissue. Stem Cells. 2007;25(5):1270-8.

18. Aydin O, Karaca G, Pehlivanli F. Platelet-Rich Plasma May Offer a New Hope in Suppressed Wound Healing When Compared to Mesenchymal Stem Cells. 2018;7(6).

19. Del Pino-Sedeno T, Trujillo-Martin MM, Andia I, Aragon-Sanchez J, Herrera-Ramos E, Iruzubieta Barragan FJ, et al. Platelet-rich plasma for the treatment of diabetic foot ulcers: A meta-analysis. Wound Repair Regen. 2019;27(2):170-82.

20. Stessuk T, Puzzi MB, Chaim EA, Alves PC, de Paula EV, Forte A, et al. Platelet-rich plasma (PRP) and adipose-derived mesenchymal stem cells: stimulatory effects on proliferation and migration of fibroblasts and keratinocytes in vitro. Arch Dermatol Res. 2016;308(7):511-20.

21. Van Pham P, Bui KH, Ngo DQ, Vu NB, Truong NH, Phan NL, et al. Activated platelet-rich plasma improves adipose-derived stem cell transplantation efficiency in injured articular cartilage. Stem Cell Res Ther. 2013;4(4):91.

22. Mahmoudian-Sani MR, Rafeei F, Amini R, Saidijam M. The effect of mesenchymal stem cells combined with platelet-rich plasma on skin wound healing. J Cosmet Dermatol. 2018;17(5):650-9.

23. Watanabe N, Ohashi K, Tatsumi K, Utoh R, Shim IK, Kanegae K, et al. Genetically modified adipose tissue-derived stem/stromal cells, using simian immunodeficiency virus-based lentiviral vectors, in the treatment of hemophilia B. Hum Gene Ther. 2013;24(3):283-94.

24. Sonnleitner D, Huemer P, Sullivan DY. A simplified technique for producing platelet-rich plasma and platelet concentrate for intraoral bone grafting techniques: a technical note. Int J Oral Maxillofac Implants. 2000;15(6):879-82.

25. Jian MY, Alexeyev MF, Wolkowicz PE, Zmijewski JW, Creighton JR. Metformin-stimulated AMPKalpha1 promotes microvascular repair in acute lung injury. Am J Physiol Lung Cell Mol Physiol. 2013;305(11):L844-55.

26. Brem H, Tomic-Canic M. Cellular and molecular basis of wound healing in diabetes. J Clin Invest. 2007;117(5):1219-22. 
27. Gentile P, Sterodimas A, Pizzicannella J, Dionisi L, De Fazio D, Calabrese C, et al. Systematic Review: Allogenic Use of Stromal Vascular Fraction (SVF) and Decellularized Extracellular Matrices (ECM) as Advanced Therapy Medicinal Products (ATMP) in Tissue Regeneration. International journal of molecular sciences. 2020;21(14).

28. Gentile P, Sterodimas A. Adipose-derived stromal stem cells (ASCs) as a new regenerative immediate therapy combating coronavirus (COVID-19)-induced pneumonia. Expert Opin Biol Ther. 2020;20(7):711-6.

29. Gentile P, Garcovich S. Concise Review: Adipose-Derived Stem Cells (ASCs) and Adipocyte-Secreted Exosomal microRNA (A-SE-miR) Modulate Cancer Growth and proMote Wound Repair. Journal of clinical medicine. 2019;8(6).

30. De Angelis B, D'Autilio M, Orlandi F, Pepe G, Garcovich S, Scioli M, et al. Wound Healing: In Vitro and In Vivo Evaluation of a Bio-Functionalized Scaffold Based on Hyaluronic Acid and Platelet-Rich Plasma in Chronic Ulcers. Journal of clinical medicine. 2019;8(9).

31. Gentile P, Garcovich S. Systematic Review-The Potential Implications of Different Platelet-Rich Plasma (PRP) Concentrations in Regenerative Medicine for Tissue Repair. International journal of molecular sciences. 2020;21(16).

32. Gentile P, Calabrese C, De Angelis B, Dionisi L, Pizzicannella J, Kothari A, et al. Impact of the Different Preparation Methods to Obtain Autologous Non-Activated Platelet-Rich Plasma (A-PRP) and Activated Platelet-Rich Plasma (AA-PRP) in Plastic Surgery: Wound Healing and Hair Regrowth Evaluation. International journal of molecular sciences. 2020;21(2).

33. Cervelli V, Bocchini I, Di Pasquali C, De Angelis B, Cervelli G, Curcio C, et al. P.R.L. platelet rich lipotransfert: our experience and current state of art in the combined use of fat and PRP. BioMed research international. 2013;2013:434191.

34. Stessuk T, Puzzi M, Chaim E, Alves P, de Paula E, Forte A, et al. Platelet-rich plasma (PRP) and adipose-derived mesenchymal stem cells: stimulatory effects on proliferation and migration of fibroblasts and keratinocytes in vitro. Archives of dermatological research. 2016;308(7):511-20.

35. Okonkwo UA, DiPietro LA. Diabetes and Wound Angiogenesis. International journal of molecular sciences. 2017;18(7).

36. Hoeben A, Landuyt B, Highley MS, Wildiers H, Van Oosterom AT, De Bruijn EA. Vascular endothelial growth factor and angiogenesis. Pharmacol Rev. 2004;56(4):549-80.

37. Bao P, Kodra A, Tomic-Canic M, Golinko MS, Ehrlich HP, Brem H. The role of vascular endothelial growth factor in wound healing. J Surg Res. 2009;153(2):347-58.

38. Hu S, Lan C. High-glucose environment disturbs the physiologic functions of keratinocytes: Focusing on diabetic wound healing. J Dermatol Sci. 2016;84(2):121-7.

39. Chen Z, Han Z. STAT3: a critical transcription activator in angiogenesis. Med Res Rev. 2008;28(2):185-200.

40. Ong HT, Dilley RJ. Novel non-angiogenic role for mesenchymal stem cell-derived vascular endothelial growth factor on keratinocytes during wound healing. Cytokine Growth Factor Rev. 2018;44:69-79. 
41. Gallagher KA, Liu ZJ, Xiao M, Chen H, Goldstein LJ, Buerk DG, et al. Diabetic impairments in NOmediated endothelial progenitor cell mobilization and homing are reversed by hyperoxia and SDF-1 alpha. J Clin Invest. 2007;117(5):1249-59.

\section{Figures}

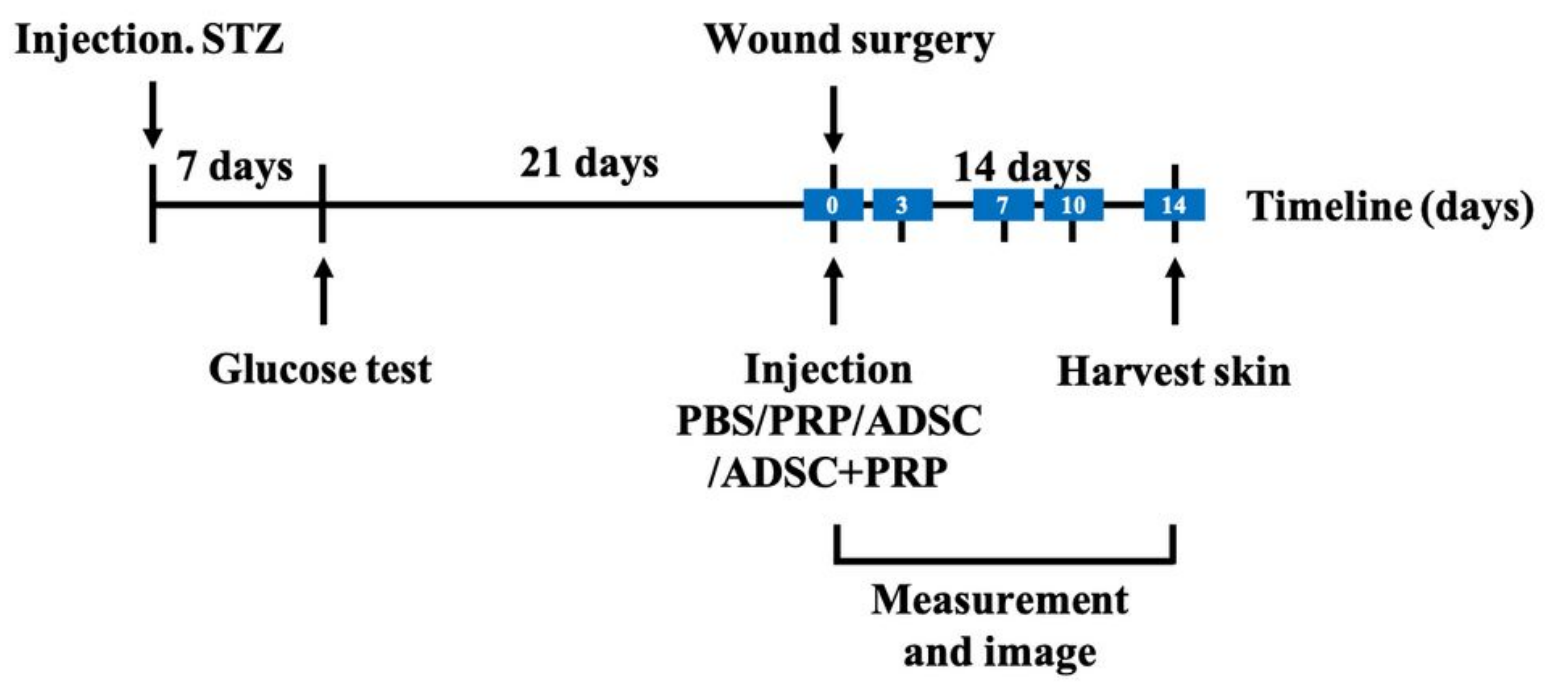

Figure 1

Timeline of the experimental design. PBS, PRP, ADSCs or ADSC-PRP was injected into the wound area, and the wound size was measured and digitally photographed each day until day 14 . 
A
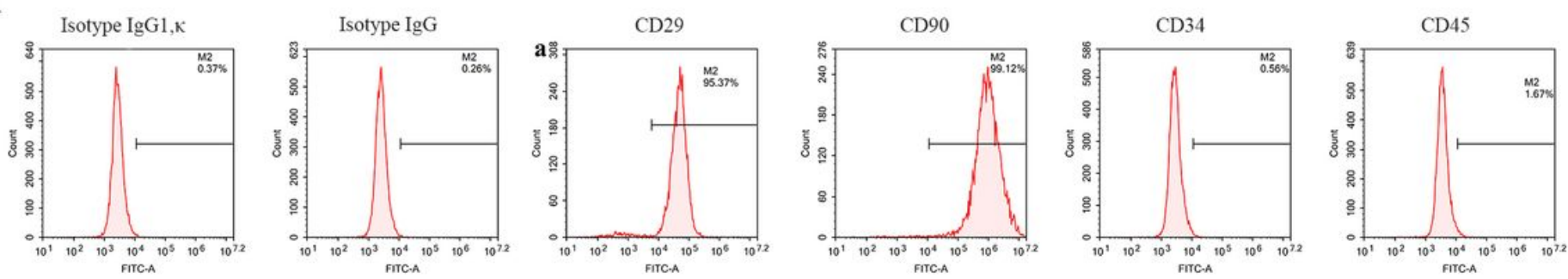

$\mathrm{B}$
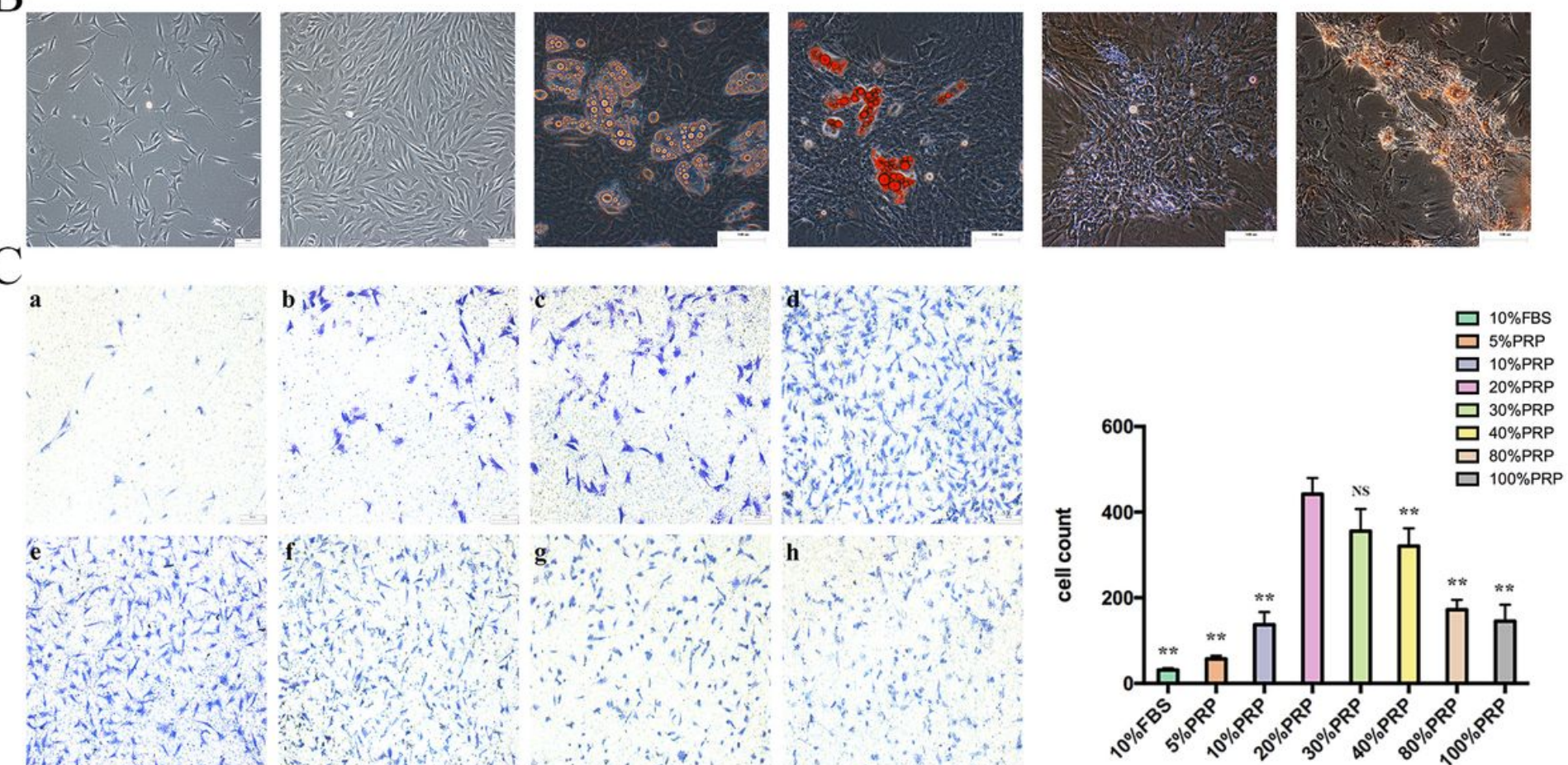

Figure 2

Characterization of ADSCs and the optimal concentration of PRP for combination with ADSCs. (A) Immunophenotypical analysis of ADSCs by flow cytometry. Passage 3 ADSCs, which were positive for CD29 and CD99 and negative for CD34, expressed typical MSC surface antigens. (B) Multipotent differentiation properties of ADSCs. Passage 3 ADSCs were cultured in adipogenic and osteogenic differentiation media for 21 days. Then, differentiation was evaluated by staining lipid droplets with oil red 0 (adipogenic, right), and calcium nodules were detected by Alizarin red staining (osteogenic, left). Representative images of 3 independent experiments are shown. Scale bars $=100 \mu \mathrm{m}$. (C) Migration efficiency was evaluated by crystal violet staining and microscopy and quantified with ImageJ. The analysis generally suggested that the optimal concentration of PRP in combination with ADSCs was $20 \%$, with efficiencies ranging from 5 to $100 \%$. All experiments were performed in triplicate and were repeated three times to confirm the findings $(* P<0.05)$. 


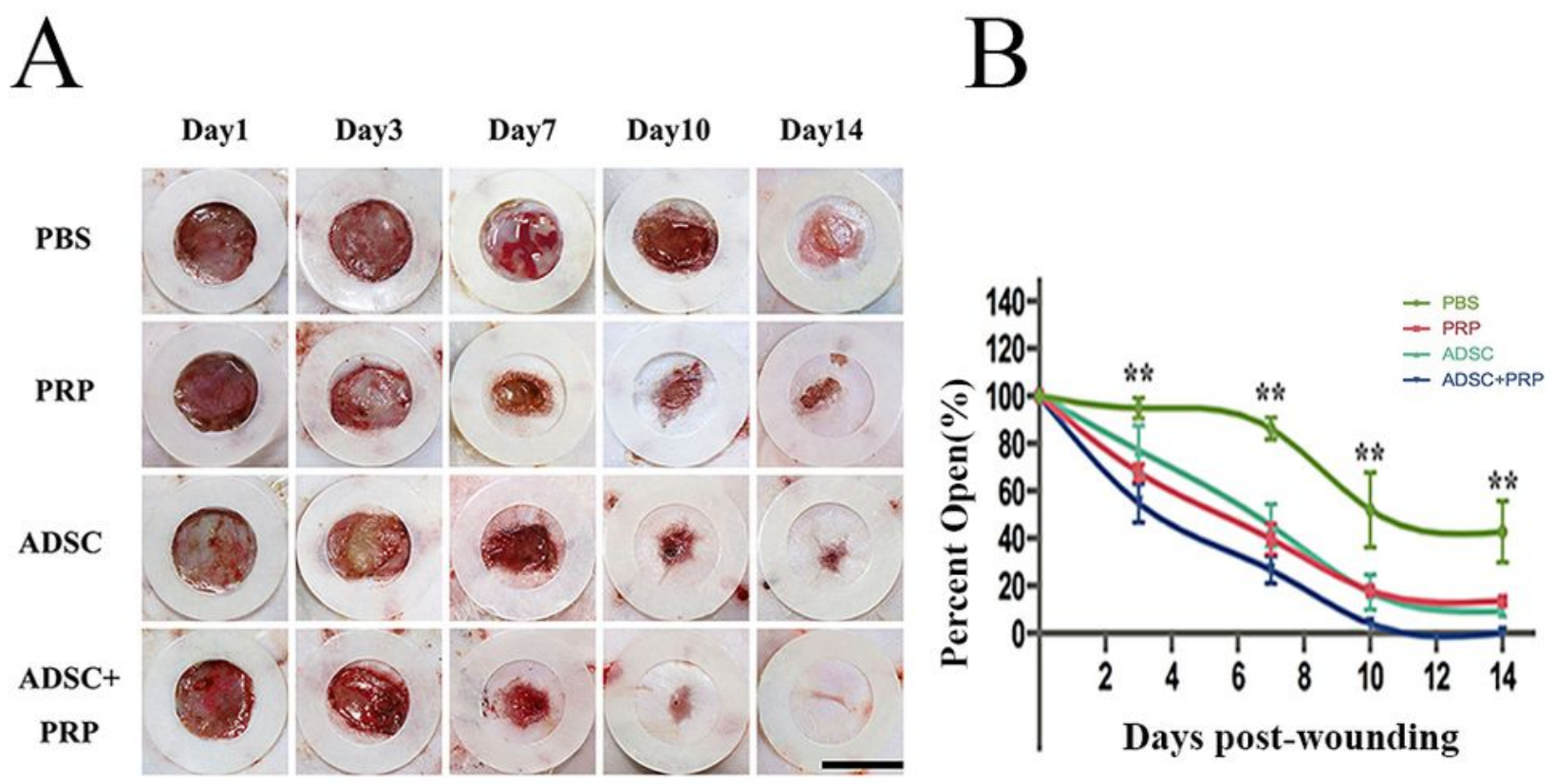

$\mathrm{C}$

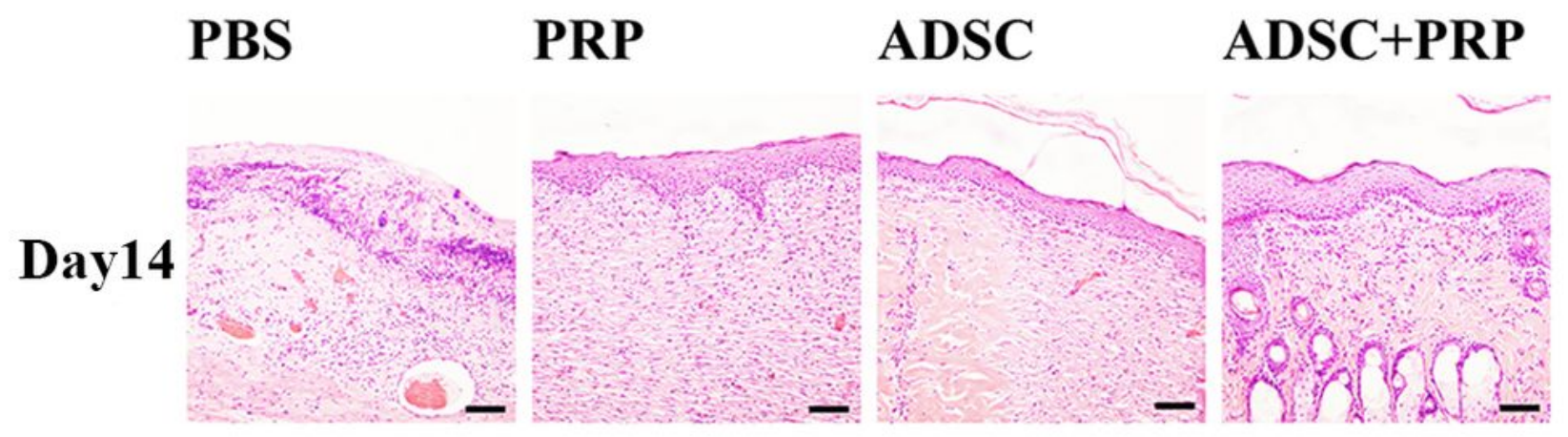

$\mathrm{D}$

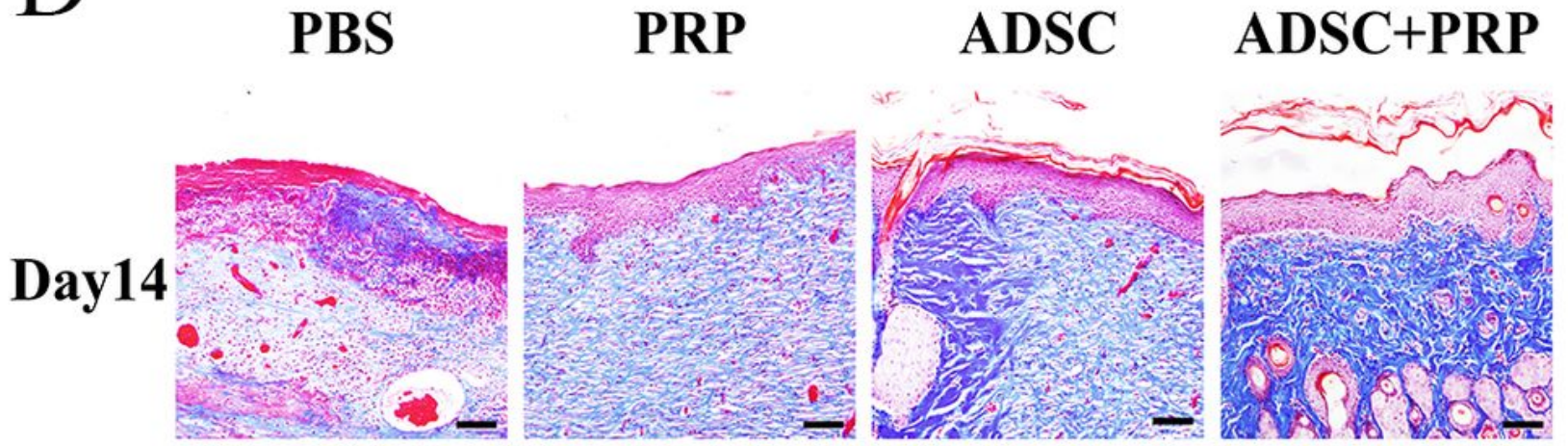

Figure 3

Wound healing in a diabetic rat excisional wound model and histology of wound beds posttreatment. (A) Representative images of the wounds show that compared to PBS, PRP, and ADSCs, treatment with ADSC-PRP accelerated wound healing, and full epithelialization was observed by day 10 . Scale bar $=1 \mathrm{~cm}$.

(B) The wound closure percentage was evaluated postwounding. The ADSC-PRP group exhibited significantly accelerated wound healing compared to that exhibited by the other groups $\left({ }^{*}<<0.05\right)$. (C) 
Hematoxylin and eosin staining on day 14 postwounding showed a thicker epidermis and increased appendages in the dermis of the ADSC-PRP group compared with the other groups. Scale bars $=50 \mu \mathrm{m}$.(D) Collagen deposition in the dermis was visualized with Masson's trichrome staining and microscopy. The analysis generally demonstrated that compared to PBS, PRP, and ADSCs, treatment with ADSC-PRP promoted epithelialization. Scale bars $=50 \mu \mathrm{m}\left({ }^{\star} \mathrm{P}<0.05 ; \mathrm{n}=3\right.$ wounds per group).

\section{A \\ Day3}

PBS

PRP

ADSC

ADSC+PRP
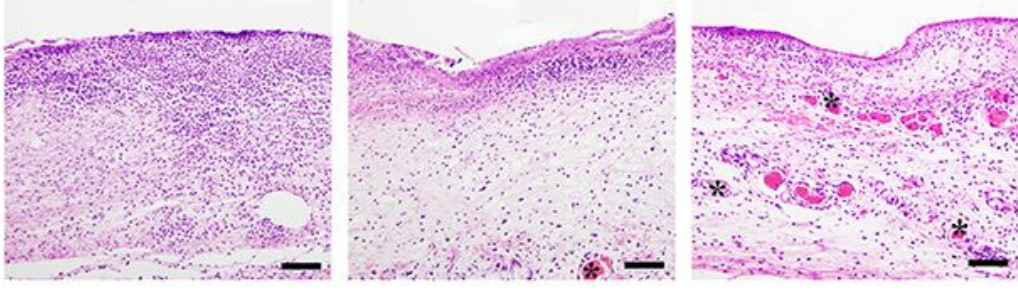

B

PBS

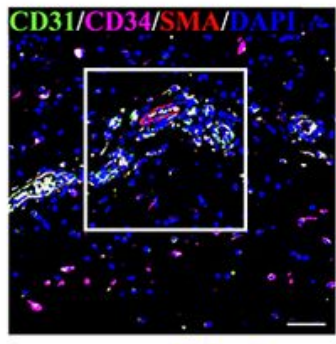

Day7

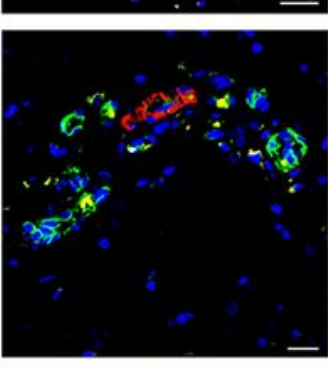

Day7
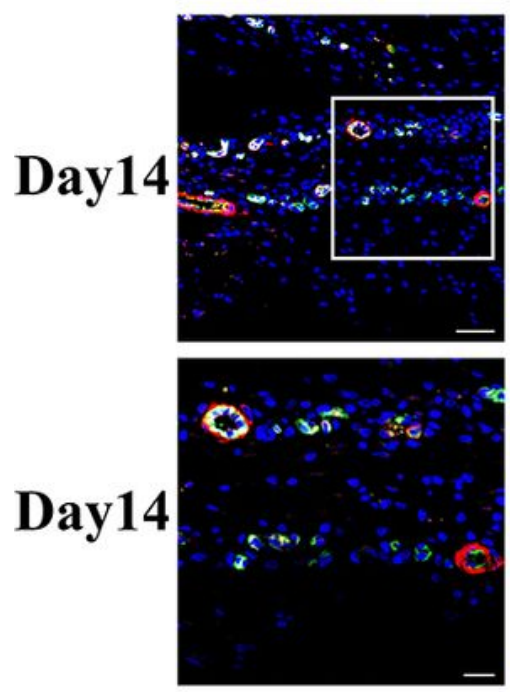

PRP
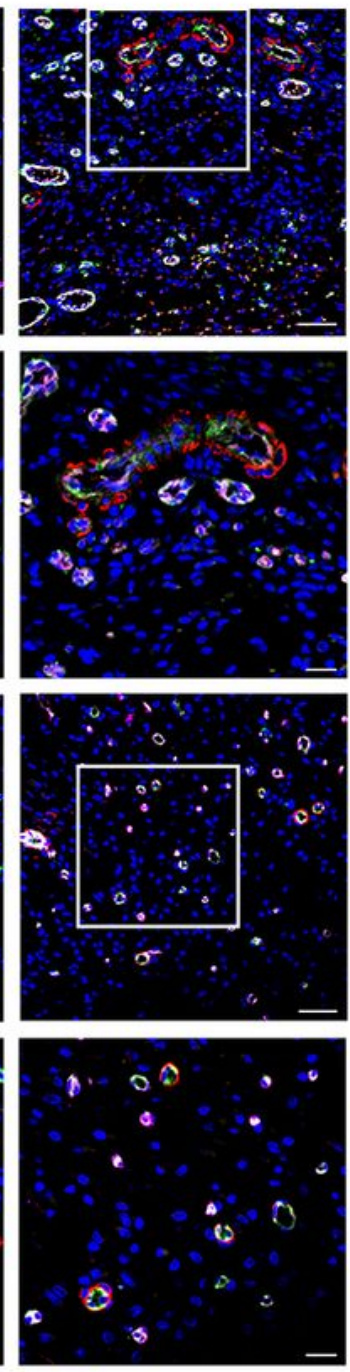

ADSC
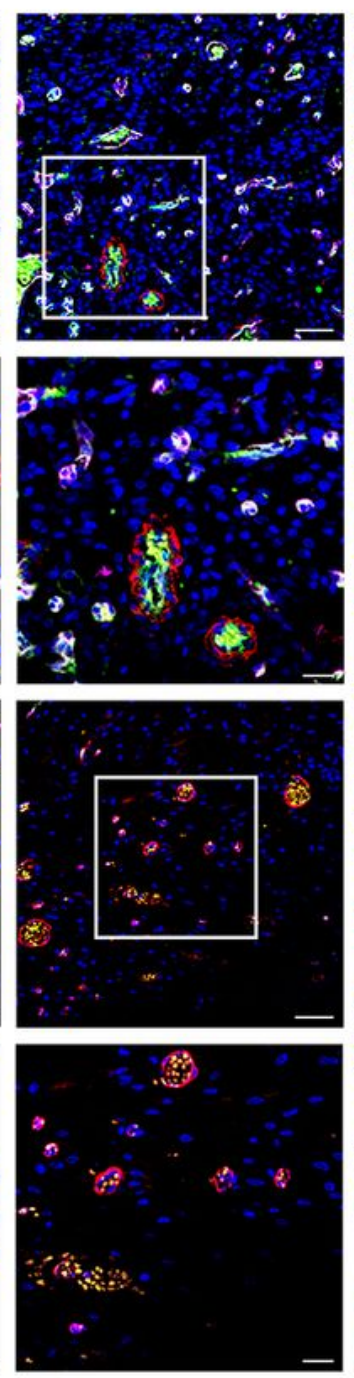

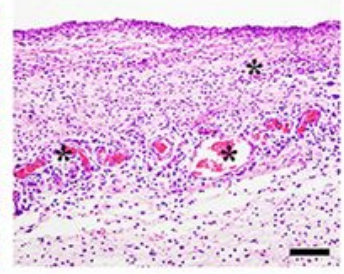

ADSC+PRP


Figure 4 
Evaluation of blood vessel formation. (A) Early angiogenesis in the wound beds was visualized on day 3 postwounding by hematoxylin and eosin staining. Staining showed an increased number of blood vessels forming in the wound in the ADSC-PRP group compared with the other groups. The black arrows indicate blood vessels. Scale bars $=50 \mu \mathrm{m}$. (B) Representative images of tissue sections from diabetic rats immunostained for CD31 (green), CD34 (pink), and a-SMA (red) and quantification of (C) the capillary density and the proportion of CD31+, CD34+, and a-SMA+ cells 7 and 14 days postwounding (200X, scale bars $=50 \mu \mathrm{m} ; 400 \mathrm{X}$, scale bars $=20 \mu \mathrm{m} ; * \mathrm{P}<0.05 ; \mathrm{n}=3$ wounds per group).



C

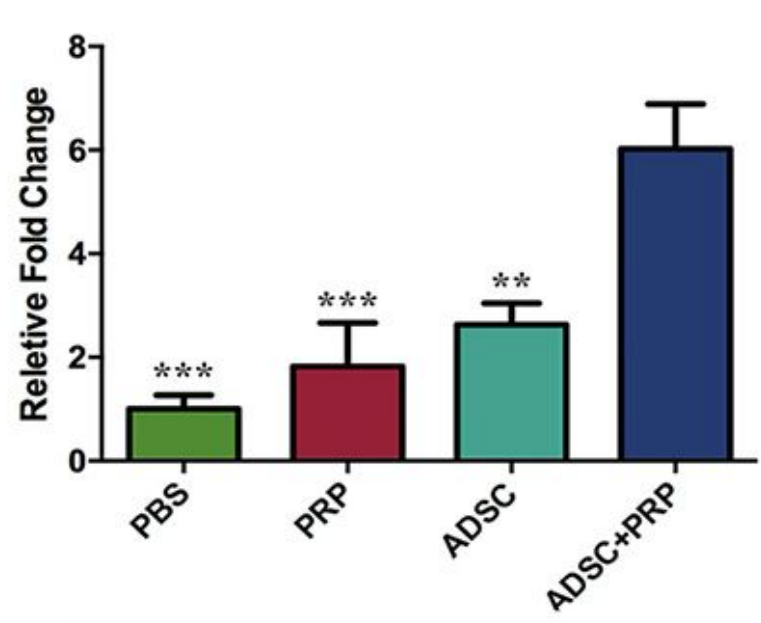

PBS PRP ADSC ADSC+

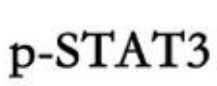

PBS

GAPDH

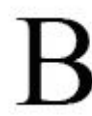

EC

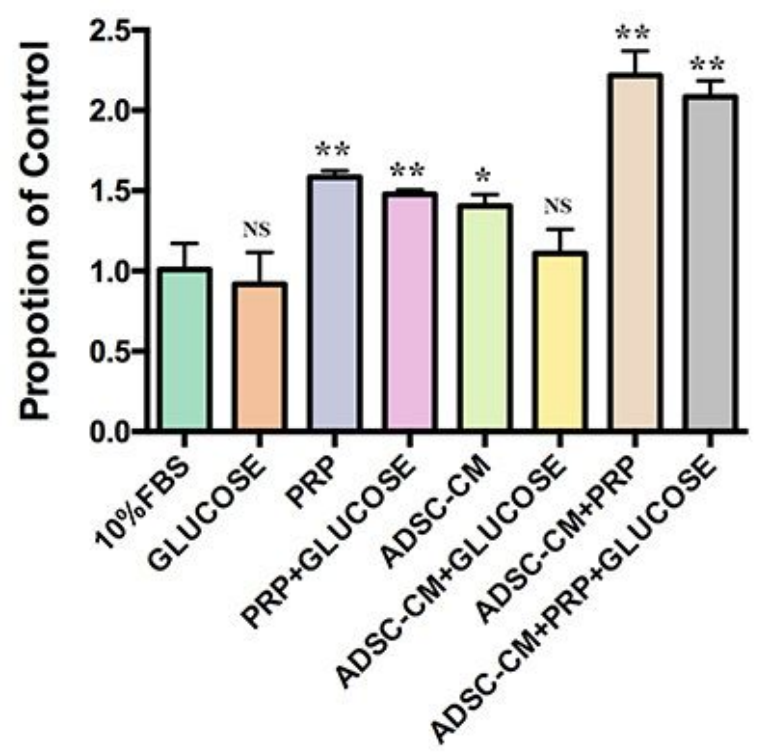

\section{PBS \\ PRP \\ ADSC \\ ADSC+PRP}



Figure 5

Effects of ADSC-PRP in promoting angiogenesis and vasculogenesis. (A) ELISA of VEGF in skin tissue lysates postwounding showed higher levels of VEGF protein production in the wounds of the ADSC-PRP group than those of the other groups. (B) Stimulation of endothelial cell proliferation by ADSC-CM and 
PRP. ADSC-PRP significantly enhanced the proliferation of endothelial cells in high-glucose medium. (C) Protein expression levels of p-STAT3 on wound tissues were detected by Western blot analysis. (D) Representative images of tissue sections from diabetic rats immunostained for SDF-1 14 days postwounding (400X, scale bars $=20 \mu \mathrm{m} ;{ }^{*} \mathrm{P}<0.05 ; \mathrm{n}=3$ wounds per group).


EC Proliferation

- PROMOTING ANGIOGENESIS OF DIABETIC WOUND

- ENHANCING THE PROLIFERATION OF EC IN A HIGH-SUGAR

ENVIRONMENT

Figure 6

Graphical abstract Graph showing the combined application of ADSC and PRP could enhance diabetic wound healing. 